\title{
ARC-SMOOTHNESS AND CONTRACTIBILITY IN WHITNEY LEVELS
}

\author{
ALEJANDRO ILLANES
}

(Communicated by James E. West)

\begin{abstract}
Let $X$ be a continuum. Let $2^{x}$ (resp., $C(X)$ ) be the space of all nonempty closed subsets (resp., subcontinua) of $X$. In this paper we prove that if $X$ is an arc-smooth continuum, then there exists an admissible Whitney map $\mu: 2^{x} \rightarrow \mathbf{R}$ such that $\mu \mid C(X): C(X) \rightarrow \mathbf{R}$ is admissible and for every $t \in(0, \mu(X)), \mu^{-1}(t)$ and $(\mu \mid C(X))^{-1}(t)$ are arc-smooth. This answers a question by J. T. Goodykoontz, Jr. Also we give an example of a contractible continuum $X$ such that, for every Whitney map $v: C(X) \rightarrow \mathbf{R}$ there exists $t \in(0, v(X))$ such that $v^{-1}(t)$ is not contractible.
\end{abstract}

\section{INTRODUCTION}

A continuum is a nondegenerate compact connected metric space. The hyperspaces of a continuum $X$ are the spaces $2^{x}=\{A \subset X: A$ is nonempty and closed in $X\}$ and $C(X)=\left\{A \in 2^{x}: A\right.$ is connected $\}$ metrized with the Hausdorff metric $H$. We identify $\{\{x\}: x \in X\} \subset C(X) \subset 2^{x}$ with $X$. A Whitney map for a hyperspace $\mathscr{H}$ of $X$ is a continuous function $\mu: \mathscr{H} \rightarrow \mathbf{R}$ ( $\mathbf{R}$ is the real line) such that $\mu(\{x\})=0$ for each $x \in X$ and if $A \subset B \neq A$, then $\mu(A)<\mu(B)$. If $\mu$ is a Whitney map for $C(X)$ and $0 \leq t<\mu(X)$, then $\mu^{-1}(t)$ is called a Whitney level; if $0<t<\mu(X)$, then $\mu^{-1}(t)$ is called a positive Whitney level. A topological property $P$ is called a Whitney property provided that whenever a continuum $X$ has property $P$, so does $\mu^{-1}(t)$ for each Whitney map for $C(X)$ and $0 \leq t<\mu(X)$. A Whitney map $\mu$ is called an admissible Whitney map for $\mathscr{H}$ [4] provided that there exists a continuous homotopy $F: \mathscr{H} \times[0,1] \rightarrow \mathscr{H}$ satisfying:

(a) For all $A \in \mathscr{H}, F(A, 1)=A$ and $F(A, 0) \in X$.

(b) If $\mu(F(A, t))>0$ for some $A \in \mathscr{H}$ and $t \in[0,1]$, then $\mu(F(A, s))<$ $\mu(F(A, t))$ whenever $0 \leq s<t$.

Let $p \in X$. Then $X$ is arc-smooth at $p$ ([1] and [2]) provided there exists a continuous function $\alpha: X \rightarrow C(X)$ satisfying:

(a) $\alpha(p)=\{p\}$.

Received by the editors July 28,1988 and, in revised form, August 11, 1989.

1980 Mathematics Subject Classification (1985 Revision). Primary 54B20. 
(b) For each $x \in X-\{p\}, \alpha(x)$ is an arc from $p$ to $x$.

(c) If $x \in \alpha(y)$, then $\alpha(x) \subset \alpha(y)$.

(The arc $\alpha(y)$ will be denoted by $[p, y]$ ).

In [5], J. Krasinkiewicz and S. B. Nadler, Jr. asked whether contractibility is a Whitney property. This question was answered negatively by A. Petrus in [7]. She showed that if $X$ is a 2-cell, then it is possible to define a Whitney map $\mu: C(X) \rightarrow \mathbf{R}$ such that for certain $t \in(0, \mu(X)), \mu^{-1}(t)$ is not contractible. Since arc-smoothness is a special type of contractibility, this example also showed that arc-smoothness is not a Whitney property. In [3], J. T. Goodykoontz, Jr. extended a previous result by Petrus giving sufficient conditions for a Whitney map to have contractible Whitney levels, and he posed the following question: Let $X$ be an arc-smooth continuum. Does there exist some Whitney map $\mu$ for $C(X)$ such that for each $t \in(0, \mu(X)), \mu^{-1}(t)$ is arc-smooth?

In this paper we answer this question affirmatively by proving that if $X$ is an arc-smooth continuum, then there exists a Whitney map $\mu: 2^{x} \rightarrow \mathbf{R}$ such that $\mu^{-1}(t)$ and $(\mu \mid C(X))^{-1}(t)$ are arc-smooth continua for every $t \in(0, \mu(X))$, and $\mu$ and $\mu \mid C(X)$ are admissible. Then arc-smoothness is a sufficient condition for $C(X)$ and $2^{x}$ to have admissible Whitney maps (compare with [3, $2.13,2.14,2.15$, and 2.16]).

In the second part of this paper we show that an analogous construction changing arc-smoothness to contractibility is not possible. We give an example of a contractible continuum $X$ such that if $v: C(X) \rightarrow \mathbf{R}$ is a Whitney map, then there exists $t \in(0, v(X))$ such that $v^{-1}(t)$ is not contractible.

\section{Construction of $\mu$}

Throughout this section we suppose that $X$ is arc-smooth at $p$. Then [2, Theorem 1-4-A] $X$ admits a metric $d$ which is radially convex at $p$ (that means that whenever $x, y \in X$ and $x \in[p, y]$, then $d(x, y)=d(p, x)+d(x, y))$. We suppose that $\sup \{d(p, x): x \in X\}=1$. Let $I$ denote the unit interval $[0,1]$.

We consider the map $h_{d}: X \times I \rightarrow X$ defined in [3, p. 144]. That is,

$$
h_{d}(x, t)= \begin{cases}x & \text { if } d(p, x) \leq 1-t \\ \text { the unique } y \in[p, x] & \text { such that } d(p, y)= \\ 1-t & \text { if } d(p, x) \geq 1-t\end{cases}
$$

This map has the following properties:

(1) For each $x \in X, h_{d}(x, 0)=x$.

(2) For each $x \in X, h_{d}(x, 1)=p$.

(3) For each $x \in X$ and $s, t \in I, h_{d}\left(h_{d}(x, s), t\right)=h_{d}(x,\{\max s, t\})$.

Define $G: 2^{x} \times I \rightarrow 2^{x}$ by $G(A, t)=\left\{h_{d}(a, t): a \in A\right\}=h_{d}(A \times\{t\})$.

The following lemma is easy to prove. 
Lemma 1.1. $G$ has the following properties:

(a) $G$ is continuous.

(b) $G \mid C(X) \times I: C(X) \times I \rightarrow C(X)$ and $g \mid X \times I: X \times I \rightarrow X$.

(c) $G(A, 0)=A$ and $G(A, 1)=\{p\}$ for every $A \in 2^{x}$.

(d) $G(G(A, s), t)=G(A, \max \{s, t\})$.

Define $l: 2^{x} \rightarrow I$ by $l(A)=\sup \{d(p, a): a \in A\}$.

Lemma 1.2. $l$ has the following properties:

(a) $l$ is continuous.

(b) $l(G(A, s))=\min \{1-s, l(A)\}$ for every $(A, s) \in 2^{x} \times I$.

(c) $s \leq t$ implies that $l(G(A, s)) \geq l(G(A, t))$.

(d) $G(A, 1-l(A))=A$ for every $A \in 2^{x}$.

Proof. We only prove (b). Let $(A, s) \in 2^{x} \times I$. Notice that if $d\left(p, h_{d}(a, s)\right) \leq$ $1-s$ for all $a \in A$, then $l(G(A, s)) \leq 1-s$. If $l(A) \leq 1-s$, then $h_{d}(a, s)=a$ for each $a \in A$. So $A=G(A, s)$. And if $l(A) \geq 1-s$, take $a_{0} \in A$ such that $l(A)=d\left(p, a_{0}\right)$. Then $d\left(p, h_{d}\left(a_{0}, s\right)\right) \geq 1-s$. So $l(G(A, s)=1-s$.

Fix a Whitney map $v: 2^{x} \rightarrow I$ with $v(X)=1$, and define $\mu: 2^{x} \rightarrow I$ by $\mu(A)=\int_{1-l(A)}^{1} v(G(A, t)) d t$.

\section{Theorem 1.3.}

(a) $\mu$ is a Whitney map.

(b) $s \leq r$ implies that $\mu(G(A, s)) \geq \mu(G(A, r))$.

(c) $(\mu \mid C(X))^{-1}(t)$ and $\mu^{-1}(t)$ are arc-smooth continua for all $t \in(0, \mu(X))$.

(d) $\mu$ and $\mu \mid C(X)$ are admissible.

Proof. (a) To see that $\mu$ is continuous, let $\varepsilon>0$. Take $\delta>0$ such that $\varepsilon>\delta$ and $H(A, B)<\delta$ implies that $|l(A)-l(B)|<\varepsilon$ and $|v(G(A, t))-v(G(B, t))|<$ $\varepsilon$ for every $t \in I$. Let $A, B \in 2^{x}$ be such that $H(A, B)<\delta$. Suppose, for example, that $l(A) \leq l(B)$. Then

$$
\begin{aligned}
|\mu(A)-\mu(B)| & \leq \int_{1-l(A)}^{1}|v(G(A, t))-v(G(B, t))| d t+\int_{1-l(B)}^{1-l(A)} v(G(B, t) d t \\
& <\varepsilon l(A)+(l(B)-l(A))<2 \varepsilon .
\end{aligned}
$$

Hence $\mu$ is continuous.

Now, take $A, B \in 2^{x}$ such that $A \subset B \neq A$. Then $l(A) \leq l(B)$ and $v(G(A, t)) \leq v(G(B, t))$ for all $t \in I$. If $l(A)=l(B)$, then $v(G(A, 1-l(A)))=$ $v(A)<v(B)=v(G(B, 1-l(A)))$. Thus $\mu(A)<\mu(B)$. If $l(A)<l(B)$, since $0<v(B)=v(G(B, 1-l(B)))$, we have that $\int_{1-l(B)}^{1-l(A)} v(G(B, t)) d t>0$. Therefore $\mu(B)>\mu(A)$. Hence $\mu$ is a Whitney map.

(b) Let $s \leq r$. Property 1.2(b) implies that $r \leq 1-l(G(A, s))$. Then Property $1.1(\mathrm{~d})$ implies that $\mu(G(A, r))=\int_{1-l(G(A, r))}^{1} G(A, t) d t$. Thus, by $1.2(\mathrm{c})$ we have $\mu(G(A, s)) \geq \mu(G(A, r))$. 
(c) Since $\mu(A)=\mu(G(A, 0)) \geq \mu(G(A, s))$ for every $s \in I, \mu \mid C(X)$ satisfies the hypothesis of Theorem 5.2 in [3]. Then $(\mu \mid C(X))^{-1}$ is arc-smooth for all $t \in(0, \mu(X))$. It is easy to check that Theorem 5.2 in [3] also holds, changing $C(X)$ to $2^{x}$. So $\mu^{-1}(t)$ is arc-smooth for all $t \in(0, \mu(X))$.

(d) Define $F: 2^{x} \times I \rightarrow 2^{x}$ by $F(A, t)=G(A, t l(A)+1-l(A))$. Then $F$ is continuous, $F(A, 0)=A$ and $F(A, 1)=\{p\}$ for each $A \in 2^{x}$, and $F \mid(C(X) \times I): C(X) \times I \rightarrow C(X)$. Suppose that $\mu(F(A, t))>0$ and take $s \in(t, 1]$. Since $F(A, t) \neq\{p\}$, we have $l(A)>0$. Set $t_{1}=t l(A)+1-l(A)$ and $s_{1}=s l(A)+1-l(A)$. Then $0 \leq l\left(G\left(A, s_{1}\right)\right)=1-s_{1}<1-t_{1}=l\left(G\left(A, t_{1}\right)\right)$. Since $G\left(A, t_{1}\right)=F(A, t)$ has more than one point, it follows that $\mu(F(A, t))>$ $\mu(F(A, s))$. Hence $\mu$ and $\mu \mid C(X)$ are admissible.

\section{THE EXAMPLE}

Let $\mathbf{R}^{3}$ be the Euclidean 3-dimensional space. Given $p, q \in \mathbf{R}^{3}$, we will denote by $\overline{p q}$ the segment joining $p$ and $q$. If $A, B \subset \mathbf{R}^{3}$ and $\alpha$ is a real number, define $A+B=\left\{p+q \in \mathbf{R}^{3}: p \in A\right.$ and $\left.q \in B\right\}, \alpha A=\left\{\alpha p \in \mathbf{R}^{3}: p \in\right.$ $A\}, \mathscr{C}(A, B)=\bigcup\{\overline{p q}: p \in A$ and $q \in B\}$ and if $\alpha \in I, \mathscr{C}(A, B, \alpha)=\{t p+$ $(1-t) q \in \mathbf{R}^{3}: p \in A, q \in B$, and $\left.0 \leq t \leq \alpha\right\}$. Then $\mathscr{C}(A, B, 1)=\mathscr{C}(A, B)$, $\mathscr{C}(A, B, 0)=B$, and if $D$ is a fixed convex continuum, $D \subset \mathbf{R}^{3}$, then the function $(A, B, \alpha) \rightarrow \mathscr{C}(A, B, \alpha)$ from $2^{D} \times 2^{D} \times I$ into $2^{D}$ is continuous.

Let $\mathbf{N}$ denote the set of positive integers. For $n \in \mathbf{N}$, set $a_{n}=1 / 2^{n}$. Define $J=\{(n, m) \in(\mathbf{N} \cup\{0\}) \times(\mathbf{N} \cup\{0\}): 0<n<m$ or $m=0<n$ or $m=0=n\}$.

Set $S_{0}=\left\{(x, y, 0) \in \mathbf{R}^{3}: x^{2}+y^{2}=1\right\}$ and $a_{0}=0$. For $n \in \mathbf{N}$, set $S_{2 n}=$ $\left\{(x, y, 0) \in \mathbf{R}^{3}: x^{2}+y^{2}=\left(1+a_{2 n}\right)^{2}\right.$ and $\left.\left\|(x, y, 0)-\left(0,1+a_{2 n}, 0\right)\right\| \geq a_{2 n}\right\}$ and $S_{2 n-1}=\left\{(x, y, 0) \in \mathbf{R}^{3}: x^{2}+y^{2}=\left(1+a_{2 n-1}\right)^{2}\right.$ and $\|(x, y, 0)-(0,-1-$ $\left.\left.a_{2 n-1}, 0\right) \| \geq a_{2 n-1}\right\}$. Then $S_{0}, S_{1}, S_{2}, \ldots$ are pairwise disjoint continua and $S_{n} \rightarrow S_{0}$.

For $(n, m) \in J$, define

$$
\begin{aligned}
L(n, m)= & \mathscr{C}\left(\left\{\left(3,0, a_{n}+a_{m}\right)\right\}+a_{n} S_{m},\left\{\left(3,0,1+a_{n}+a_{m}\right)\right\}\right) \\
& \cup \mathscr{C}\left(\left\{\left(8,0, a_{n}+a_{m}\right)\right\}+a_{n} S_{m},\left\{\left(8,0,1+a_{n}+a_{m}\right)\right\}\right) \\
& \cup \overline{\left(3+a_{n}\left(1+a_{m}\right), 0, a_{n}+a_{m}\right)\left(8-a_{n}\left(1+a_{m}\right), 0, a_{n}+a_{m}\right)}
\end{aligned}
$$

and

$$
K(n, m)=L(n, m) \cup \overline{\left(0,0, a_{n}+a_{m}\right)\left(3-a_{n}\left(1+a_{m}\right), 0, a_{n}+a_{m}\right)} .
$$

Then define $X=(\bigcup\{K(n, m):(n, m) \in J\}) \cup \overline{(0,0,0)(0,0,2)}$. Clearly, $X$ is a contractible continuum.

Theorem 2.1. If $\mu: C(X) \rightarrow \mathbf{R}$ is a Whitney map, then there exists $t \in(0, \mu(X))$ such that $\mu^{-1}(t)$ is not contractible. 
Proof. Let $\mu: C(X) \rightarrow \mathbf{R}$ be a Whitney map. Set

$$
\begin{aligned}
& D=\overline{(3,0,0)(8,0,0)}, \\
& E=D \cup \overline{(8,0,0)(8,0,1)}, \\
& F=D \cup \overline{(3,0,0)(3,0,1)},
\end{aligned}
$$

and

$$
G=\overline{(0,0,0)(8,0,0)} .
$$

Take any $t \in \mathbf{R}$ such that $\mu(D)<t<\mu(E), \mu(F)$ and $\mu(G)$. We will prove that $\mu^{-1}(t)$ is not contractible. Since $D \subset E$, there exists $H \in C(X)$ such that $\mu(H)=t$ and $D \subset H \subset E$. Suppose that $\mu^{-1}(t)$ is contractible. Then there exists a continuous function $f: \mu^{-1}(t) \times I \rightarrow \mu^{-1}(t)$ such that $f(A, 0)=A$ and $f(A, 1)=H$ for every $A \in \mu^{-1}(t)$.

Given $(n, m) \in J$ and $r \in I$, let

$$
\begin{aligned}
L(n, m, r)= & \mathscr{C}\left(\left\{\left(3,0,1+a_{n}+a_{m}\right)\right\},\left\{\left(3,0, a_{n}+a_{m}\right)\right\}+a_{n} S_{m}, r\right) \\
& \cup \mathscr{C}\left(\left\{\left(8,0,1+a_{n}+a_{m}\right)\right\},\left\{\left(8,0, a_{n}+a_{m}\right)\right\}+a_{n} S_{m}, r\right) \\
& \cup \overline{\left(3+a_{n}\left(1+a_{m}\right), 0, a_{n}+a_{m}\right)\left(8-a_{n}\left(1+a_{m}\right), 0, a_{n}+a_{m}\right)} .
\end{aligned}
$$

Then $L(n, m, 1)=L(n, m), L(n, 0,0) \rightarrow D$, and $L(n, 0,1) \rightarrow E \cup F$. So we can choose $n \in \mathbf{N}$ such that $\mu(L(n, 0,0))<t<\mu(L(n, 0,1))$. We may also assume that if $A \in C(X)$ and $\left(3+a_{n}, 0, a_{n}\right),\left(8,0,1+a_{n}\right) \in A$ or $\left(3,0,1+a_{n}\right),\left(8-a_{n}, 0, a_{n}\right) \in A$ or $\left(0,0, a_{n}\right),\left(8-a_{n}, 0, a_{n}\right) \in A$, then $\mu(A)>t$. Let $u_{0}$ be the unique element of the interval $(0,1)$ such that $\mu\left(L\left(n, 0, u_{0}\right)\right)=t$.

Since $L(n, m, 0) \rightarrow L(n, 0,0)$ and $L(n, m, 1) \rightarrow L(n, 0,1)$ when $m \rightarrow$ $\infty$, there exists $M>n$ such that, for every $m \geq M, \mu(L(n, m, 0)<t<$ $\mu(L(n, m, 1))$. Then for each $m \geq M$ there exists a unique $u_{m} \in(0,1)$ such that $\mu\left(L\left(n, m, u_{m}\right)\right)=t$. Set $C_{m}=L\left(n, m, u_{m}\right)$. The uniqueness of $u_{0}$ implies that $u_{m} \rightarrow u_{0}$ and then $C_{m} \rightarrow L\left(n, 0, u_{0}\right)$ when $m \rightarrow \infty$.

Let $C=L\left(n, 0, u_{0}\right), p_{0}=\left(3+a_{n}, 0, a_{n}\right)$, and $q_{0}=\left(8-a_{n}, 0, a_{n}\right)$. Then $p_{0}, q_{0} \in f(C, 0)-f(C, 1)$. Let $s_{0}=\max \left\{s \in I: p_{0}\right.$ and $q_{0}$ belongs to $f(C, r)$ for all $r \in[0, s]\}$. Then $s_{0}<1$. Let $L=L(n, 0,0)$ $\left(=\left(\left\{\left(3,0, a_{n}\right)\right\}+a_{n} S_{0}\right) \cup\left(\left\{\left(8,0, a_{n}\right)\right\}+a_{n} S_{0}\right) \cup\left(3+a_{n}, 0, a_{n}\right)\left(8-a_{n}, 0, a_{n}\right)\right)$, $R=L \cup\left(0,0, a_{n}\right)\left(3-a_{n}, 0, a_{n}\right) \subset K(n, 0), p_{1}=\left(3,0,1+a_{n}\right)$, and $q_{1}=$ $\left(8,0,1+a_{n}\right)$. Let $h: K(n, 0)-\left\{p_{1}, q_{1}\right\} \rightarrow R$ be the natural retraction.

The choice of $n$ and $s_{0}$ implies that $p_{1}, q_{1}$, and $\left(0,0, a_{n}\right) \notin f(C, r)$ for all $r \in\left[0, s_{0}\right]$. Then it is possible to define $g:\left[0, s_{0}\right] \rightarrow C(R)$ by $g(s)=$ $h(f(C, s)$ ) (the image under $h$ of $f(C, s)$ ).

Let $r_{0}=\max \left\{r \in\left[0, s_{0}\right]: L \subset g(s)\right.$ for all $\left.s \in[0, r]\right\}$. We will prove that $r_{0}=s_{0}$. Suppose that $r_{0}<s_{0}$. Then there exists $r_{1} \in\left(r_{0}, s_{0}\right)$ such that $L \not \subset g\left(r_{1}\right)$ and $H\left(g\left(r_{0}\right), g\left(r_{1}\right)\right)<a_{n} / 4(H$ is the Hausdorff metric for $C(X))$. Since $p_{0}, q_{0} \in g\left(r_{1}\right)$, then $\overline{p_{0} q_{0}} \subset g\left(r_{1}\right)$. So we assume, for example, that 
there exists a point $p_{2}=\left(3,0, a_{n}\right)+a_{n} z_{0} \in L-g\left(r_{1}\right)$ with $z_{0}=\left(x_{0}, y_{0}, 0\right) \in$ $S_{0}-\{(1,0,0)\}$. We analyze the case $y_{0} \geq 0$. The case $y_{0} \leq 0$ is similar.

Let $l=\overline{p_{1} p_{2}}$. Since $h^{-1}\left(p_{2}\right)=l-\left\{p_{1}\right\}$, we have $f\left(C, r_{1}\right) \cap l=\varnothing$. Given $m$ odd with $m>n$, set $z_{m}=\left(1+a_{m}\right) z_{0} \in S_{m}, p_{m}=\left(3,0, a_{n}+a_{m}\right)+a_{n} z_{m} \in$ $L(n, m)$, and $l_{m}=\overline{p_{m}\left(3,0,1+a_{n}+a_{m}\right)} \subset L(n, m)$. Then $l_{m} \rightarrow l$ when $m \rightarrow \infty$ ( $m$ odd $)$. Since $f\left(C, r_{1}\right) \cap l=\varnothing, f\left(C_{m}, r_{1}\right) \rightarrow f\left(C, r_{1}\right)$, and $\left(0,0, a_{n}\right) \notin f(C, s)$ for each $s \in\left[0, r_{1}\right]$, there exists $M_{1}>M$ such that if $m \geq M_{1}$ and $m$ is odd, then $f\left(C_{m}, r_{1}\right) \cap l_{m}=\varnothing$ and $\left(0,0, a_{n}+a_{m}\right) \notin$ $f\left(C_{m}, s\right)$ for each $s \in\left[0, r_{1}\right]$. Since $f\left(C_{m}, 0\right)=C_{m} \subset K(n, m)$, if $m \geq M_{1}$ and $m$ is odd, then $f\left(C_{m}, r_{1}\right) \subset K(n, m)-l_{m}$. Since $f\left(C_{m}, r_{1}\right) \rightarrow f\left(C, r_{1}\right)$ and $q_{0} \in f\left(C, r_{1}\right)$, there exists $M_{2} \geq M_{1}$ such that if $m \geq M_{2}$ and $m$ is odd, then $f\left(C_{m}, r_{1}\right)$ is contained in the component of $K(n, m)-l_{m}$ which has the point $\left(8-a_{n}\left(1+a_{m}\right), 0, a_{n}+a_{m}\right)$. This implies that $f\left(C, r_{1}\right)$ is contained in the closure of the component of $K(n, 0)-\left(l \cup \overline{p_{1}\left(3,-a_{n}, a_{n}\right)}\right)$, which has the point $\left(8-a_{n}, 0, a_{n}\right)$. Then $g\left(r_{1}\right)=h\left(f\left(C, r_{1}\right)\right)$ is contained in the closure of the component of $L-\left\{p_{2},\left(3,-a_{n}, a_{n}\right)\right\}$, which has the point $\left(8-a_{n}, 0, a_{n}\right)$. This is a contradiction since $y_{0} \geq 0, H\left(g\left(r_{0}\right), g\left(r_{1}\right)\right)<a_{n} / 4$, and $L \subset g\left(r_{0}\right)$. Hence $r_{0}=s_{0}$.

In particular, $L \subset g\left(s_{0}\right) \subset K(n, 0)-\left\{\left(0,0, a_{n}\right)\right\}$. Since $s_{0}<1$, it follows that there exists $s^{\prime} \in\left(s_{0}, 1\right)$ such that $p_{0}, q_{0} \in f(C, s)$ for all $s \in\left[s_{0}, s^{\prime}\right]$. This contradicts the choice of $s_{0}$ and ends the proof that $\mu^{-1}(t)$ is not contractible.

\section{BIBLIOGRAPHY}

1. J. B. Fugate, G. R. Gordh, Jr., and L. Lum, On arc-smooth continua, Topology Proc. 2 (1977), 645-656.

2. __ Arc-smooth continua, Trans. Amer. Math. Soc. 265 (1981), 545-561.

3. J. T. Goodykoontz, Jr., Arc-smoothness in hyperspaces, Topology Appl. 15 (1985), 131-150.

4. J. T. Goodykoontz, Jr. and S. B. Nadler, Jr., Whitney levels in hyperspaces of certain Peano continua, Trans. Amer. Math. Soc. 274 (1982), 671-694.

5. J. Krasinkiewicz and S. B. Nadler, Jr., Whitney properties, Fund. Math. 98 (1978), 165-180.

6. S. B. Nadler, Jr., Hyperspaces of sets, Pure Appl. Math., vol. 49, Dekker, New York, 1978.

7. A. Petrus, Contractibility of Whitney continua in $C(X)$, Gen. Topology Appl. 9 (1978), 275-288.

Instituto de Matemáticas, Circuito Exterior, Ciudad Universitaria, México 04510, Distrito Federal, Mexico 